\title{
Pair Correlations in the Two-Dimensional Fermi Gas
}

\author{
Vudtiwat Ngampruetikorn, ${ }^{1}$ Jesper Levinsen, ${ }^{1,2}$ and Meera M. Parish ${ }^{3}$ \\ ${ }^{1}$ T.C.M. Group, Cavendish Laboratory, JJ Thomson Avenue, Cambridge, CB3 0HE, United Kingdom \\ ${ }^{2}$ Aarhus Institute of Advanced Studies, Aarhus University, DK-8000 Aarhus C, Denmark \\ ${ }^{3}$ London Centre for Nanotechnology, Gordon Street, London, WC1H 0AH, United Kingdom
}

(Dated: November 1, 2018)

\begin{abstract}
We consider the two-dimensional Fermi gas at finite temperature with attractive short-range interactions. Using the virial expansion, which provides a controlled approach at high temperatures, we determine the spectral function and contact for the normal state. Our calculated spectra are in qualitative agreement with recent photoemission measurements [M. Feld et al., Nature 480, 75 (2011)], thus suggesting that the observed pairing gap is a feature of the high-temperature gas rather than being evidence of a pseudogap regime just above the superfluid transition temperature. We further argue that the strong pair correlations result from the fact that the crossover to bosonic dimers occurs at weaker interactions than previously assumed.
\end{abstract}

The two-dimensional (2D) Fermi gas with contact interactions provides a basic model for understanding pairing and superconductivity in 2D systems [1-3]. Recently, there has been a resurgence of interest in this model owing to the realization of $2 \mathrm{D}$ Fermi gases in cold-atom experiments [4-13]. In particular, there is the possibility of investigating deviations from Fermi liquid behavior at finite temperature. However, 2D systems are notoriously difficult to treat theoretically since mean-field theory is less reliable in low dimensions, while at the same time there is a dearth of exact solutions, in contrast to the case in 1D. Hence there is a need for controlled perturbative approaches that can guide theory and experiment. The virial expansion is one such controlled approach in the high temperature limit, and this will be the focus of our Letter.

Before investigating finite temperature, it is instructive to consider the ground state of the two-component $(\uparrow, \downarrow)$ Fermi gas in 2D. Here we assume that the masses and chemical potentials of each spin are equal, i.e., $m_{\uparrow}=m_{\downarrow} \equiv m$ and $\mu_{\uparrow}=\mu_{\downarrow} \equiv \mu$, respectively. Then one expects a smooth crossover from BCS pairing to BoseEinstein condensation (BEC) of tightly bound $\uparrow \downarrow$ dimers with increasing interspecies attraction. Since there is always a two-body bound state for attractive contact interactions in 2D, one can define the strength of the interaction using the two-body binding energy $\varepsilon_{B}=\hbar^{2} / m a_{2 \mathrm{D}}^{2}$, where $a_{2 \mathrm{D}}$ is the $2 \mathrm{D}$ scattering length. Thus, for a Fermi gas with total density $n=k_{F}^{2} / 2 \pi$, where $k_{F}$ is the Fermi wave vector, we have $k_{F} a_{2 \mathrm{D}} \gg 1$ in the BCS limit and $k_{F} a_{2 \mathrm{D}} \ll 1$ in the BEC limit. Note that the crossover can be driven by varying the density as well as by varying the interaction, unlike the case in 3D.

Mean-field theory has provided a qualitative understanding of the 2D BCS-BEC crossover [1,2], while recent quantum Monte Carlo (QMC) calculations have determined the ground state energy [14]. However, there is surprisingly little discussion about what constitutes the crossover point. As previously argued in the 3D case, the crossover is best defined as the point where $\mu=0$, since this can signal the disappearance of a Fermi surface and an associated change in the quasiparticle excitation spectrum $[15,16]$. Indeed, while the $s$-wave paired superfluid is characterized by a crossover, a phase transition at $\mu=0$ is predicted in the $p$-wave paired superfluid [17]. In $2 \mathrm{D}$, it is generally assumed that the crossover occurs at $\ln \left(k_{F} a_{2 \mathrm{D}}\right)=0$, which is consistent with the mean-field chemical potential, $\mu=\varepsilon_{F}-\varepsilon_{B} / 2$, being zero (where $\varepsilon_{F}=\hbar^{2} k_{F}^{2} / 2 m$ is the Fermi energy). However, it is easy to show using the QMC data from Ref. [14] that the chemical potential in fact vanishes at $\ln \left(k_{F} a_{2 \mathrm{D}}\right) \simeq 0.5$, corresponding to $n a_{2 \mathrm{D}}^{2} \simeq 0.4$. This suggests that experiments on the $2 \mathrm{D}$ Fermi gas have mainly explored the BEC side of the crossover, as we discuss below. Therefore, it is perhaps not surprising that recent radio-frequency (rf) measurements in the regime $\ln \left(k_{F} a_{2 \mathrm{D}}\right) \lesssim 0.6$ agree well with two-body theory [8].

This also has implications for the normal state above the superfluid transition temperature $T_{c}$. In particular, the highly sought-after "pseudogap" regime requires the presence of a Fermi surface [18]: while this phase is often synonymous with pairing above $T_{c}$ in the cold-atom literature, if one is to emulate the pseudogap phenomena in high temperature superconductors, then one must have a loss of spectral weight, i.e., a gap, at the Fermi surface [19]. Thus, a pairing gap above $T_{c}$ does not imply a pseudogap when $\mu<0$ and Pauli blocking is minimal.

In this Letter, we investigate the $2 \mathrm{D}$ Fermi gas at finite temperature $T$ using the virial expansion. We focus on the pair correlations as encoded in the contact [20] and the spectral function, rather than the thermodynamic properties and the equation of state, which have been determined in Ref. [21]. We find that our calculated spectra qualitatively reproduce those from recent photoemission experiments [5], and we argue that the observed pairing gap above $T_{c}$ is in the regime where $\mu<0$.

The basic idea of the virial expansion, as applied to the uniform 2D Fermi gas, can be summarised as follows. Working in the grand canonical ensemble, we define the virial coefficients $b_{j}$ such that the grand potential 
$\Omega(T, V, \mu)$ is given by (we set $\hbar=k_{B}=1$ )

$$
\Omega=-2 T V \lambda^{-2} \sum_{j \geq 1} b_{j} z^{j}
$$

where the thermal wavelength $\lambda=\sqrt{2 \pi / m T}$ and the fugacity $z=e^{\beta \mu}$. We also denote $V$ as the system area and $\beta \equiv 1 / T$. In the high temperature limit where $\lambda^{2} n \lesssim 1$, the above power series may be truncated and the thermodynamics of the system can be accurately described by only the first few virial coefficients. This usually corresponds to an expansion in small $z$, but care must be exercised when treating the Bose limit $k_{F} a_{2 \mathrm{D}} \ll 1$. In this case, $\mu \simeq-\varepsilon_{B} / 2$ at low temperatures so that $z \simeq e^{-\beta \varepsilon_{B} / 2} \rightarrow 0$ as $T \rightarrow 0$, which naively suggests that the virial expansion is valid even at zero temperature. However, as we show below, the coefficients $b_{j}$ also contain powers of $e^{\beta \varepsilon_{B} / 2}$ that cancel the contribution from the binding energy in $z$ when $j$ is even. Thus, the relevant expansion parameter is in fact $z^{(\text {Bose })}=z e^{\beta \varepsilon_{B} / 2}$, with corresponding coefficients $b_{j}^{\text {(Bose) }}=e^{-j \beta \varepsilon_{B} / 2} b_{j}$.

To proceed further, we consider the total density

$$
n=-\left.\frac{1}{V} \frac{\partial \Omega}{\partial \mu}\right|_{T, V}=2 \lambda^{-2} \sum_{j \geq 1} j b_{j} z^{j}
$$

with the density of each species given by $n_{\sigma}=n / 2$. The non-interacting part of the virial coefficient is then readily obtained from $n_{\sigma}=\sum_{\mathbf{k}} n_{F}\left(\epsilon_{\mathbf{k}}-\mu\right)$, where $n_{F}$ is the Fermi-Dirac distribution function. This yields $b_{j}^{\text {(free) }}=(-1)^{j-1} j^{-2}$ for $j \geq 1$. We obtain the second and third virial coefficients using the diagrammatic method recently introduced in 3D by Leyronas [22]. As a starting point, we write down the relation between the density and the propagator, $n_{\sigma}(\mu, T)=\sum_{\mathbf{k}} G_{\sigma}\left(\mathbf{k}, \tau=0^{-}\right)$. As usual, the full Green's function on the right-hand side is then written in terms of the bare propagators $G^{(0)}(\mathbf{k}, \tau)$. The key point is that these may be expanded in powers of $z$,

$$
\begin{aligned}
G_{\sigma}^{(0)}(\mathbf{k}, \tau) & =e^{-\left(\epsilon_{\mathbf{k}}-\mu\right) \tau}\left[-\Theta(\tau)+n_{F}\left(\epsilon_{\mathbf{k}}-\mu\right)\right] \\
& =e^{\mu \tau} \sum_{j \geq 0} G_{\sigma}^{(0, j)}(\mathbf{k}, \tau) z^{j}
\end{aligned}
$$

where the 'virial propagators' are defined as

$$
G_{\sigma}^{(0, j)}(\mathbf{k}, \tau)=\left\{\begin{array}{lll}
-\Theta(\tau) e^{-\epsilon_{\mathbf{k}} \tau} & \text { if } & j=0 \\
(-1)^{j-1} e^{-\epsilon_{\mathbf{k}} \tau-j \beta \epsilon_{\mathbf{k}}} & \text { if } & j \geq 1
\end{array}\right.
$$

This allows one to construct a perturbative expansion in $z$ for the full propagator. As an example, Fig. 1(a) depicts the diagrammatic representation of the virial expansion of the free propagator. The non-interacting virial coefficients are also easily recovered using the virial propagators above. Note that $G^{(0,0)}$ is the only retarded term, meaning it vanishes whenever $\tau<0$.

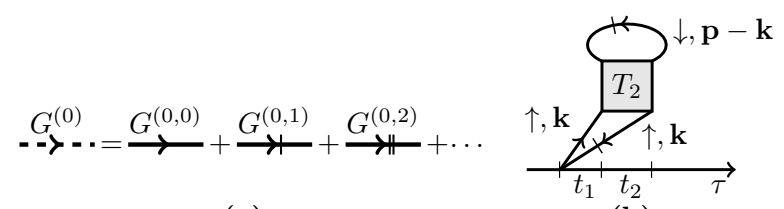

(a)

(b)

FIG. 1: (a) Diagramatic representation of the virial expansion of the bare propagator. The number of vertical dashes indicates the power of fugacity of the corresponding term in Eq. (4). (b) Second order contribution to density due to interactions. $t_{1}$ and $t_{2}$ represent intervals on the imaginary time axis.

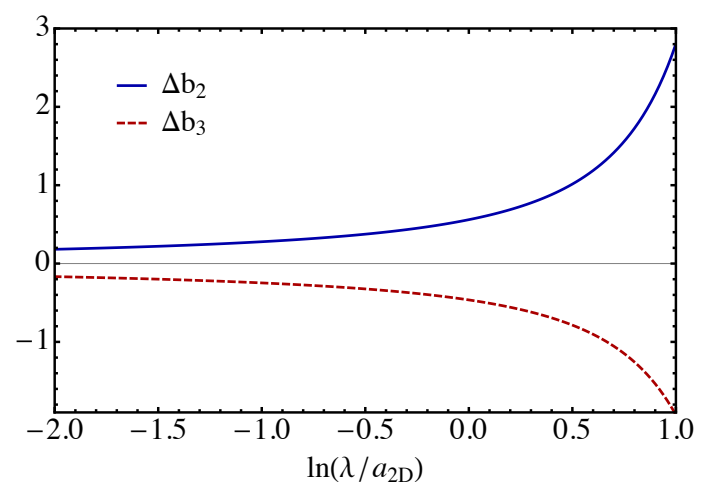

FIG. 2: The contribution from interactions to the second and third virial coefficients of the uniform 2D Fermi gas.

In order to obtain the second virial coefficient, we must calculate the term arising from interactions and contributing to the density at second order in the fugacity:

$$
\begin{aligned}
2 z^{2} \sum_{\mathbf{k p}} \int \mathrm{d} t_{1} \mathrm{~d} t_{2} T_{2}\left(\mathbf{p}, t_{2}\right) G_{\uparrow}^{(0,0)}\left(\mathbf{k}, t_{1}\right) \\
\quad \times G_{\uparrow}^{(0,1)}\left(-\mathbf{k},-t_{1}-t_{2}\right) G_{\downarrow}^{(0,1)}\left(\mathbf{p}-\mathbf{k},-t_{2}\right),
\end{aligned}
$$

as illustrated in Fig. 1(b). The factor of 2 accounts for the spin degeneracy. The $T$-matrix $T_{2}$ [33] is defined purely in terms of the retarded virial propagator $G^{(0,0)}$ and as a result $T_{2}$ is also retarded. Following manipulations of the time integrations along the lines set out in Ref. [22], we find that the second virial coefficient consists of two terms. The first arises from the dimer pole of the $T$ matrix and is given by $e^{\beta \varepsilon_{B}}$, while the second comes from the scattering states. We then obtain

$$
\Delta b_{2}=e^{\beta \varepsilon_{B}}-\int_{0}^{\infty} \frac{\mathrm{d} p}{p} \frac{2 e^{-\beta p^{2} / m}}{\pi^{2}+4 \ln ^{2}\left(p a_{2 \mathrm{D}}\right)},
$$

where we define $\Delta b_{j} \equiv b_{j}-b_{j}^{\text {(free) }}$. In the limit $T \ll \varepsilon_{B}$, the exponential term dominates as expected, since all the atoms are then bound into pairs. We see that, as the virial expansion contains terms contributing separately to the atom and dimer densities, measuring these in experiment may be used to extract the chemical potential 
and temperature, within the regime of validity of the approximation. The dimer pole of the $T$-matrix also plays a major role in the higher order coefficients $b_{j}$, contributing $e^{j \beta \varepsilon_{B} / 2}$ for even $j$ and $e^{(j-1) \beta \varepsilon_{B} / 2}$ for odd $j$. Comparing this with the factors of $e^{-\beta \varepsilon_{B} / 2}$ in $z$ discussed above, we thus expect odd terms in the expansion to tend to zero as $\beta \varepsilon_{B} \rightarrow \infty$. Beyond that, the calculation of the third virial coefficient is significantly more involved, and we refer the interested reader to Ref. [22] for the required diagrams.

Figure 2 displays the behavior of the second and third virial coefficients in the regime of interactions before $e^{\beta \varepsilon_{B}}$ dominates. We stress that the virial coefficients are functions of $\ln \left(\lambda / a_{2 \mathrm{D}}\right)$, or equivalently $\beta \varepsilon_{B}$, only. We see that the correction to the second virial coefficient due to interactions is attractive, since it increases the density at fixed $\mu$ and $T$. However, this lowest order term is expected to overestimate the attraction at lower temperatures and thus the third-order correction acts to suppress the density.

We now turn to the investigation of pair correlations, for which we first discuss the virial expansion of the contact [20] in 2D. Within the zero-range model, this quantity is given by $\mathcal{C}=2 \pi m \frac{\mathrm{d} E}{\mathrm{~d} \ln \left(a_{2 \mathrm{D}}\right)}$ [23]. The contact can also be expressed in terms of the grand potential $\Omega(T, V, \mu)$, thus giving

$$
\mathcal{C}=2 \pi m\left(\frac{\partial \Omega}{\partial \ln \left(a_{2 \mathrm{D}}\right)}\right)_{T, V, \mu}=\frac{8 \pi^{2} V}{\lambda^{4}} \sum_{j \geq 2} c_{j} z^{j}
$$

with 'contact' coefficients $c_{j}=\left[\mathrm{d} \Delta b_{j} / \mathrm{d} \ln \left(\lambda / a_{2 \mathrm{D}}\right)\right]_{T}$ (for the 3D equivalent, see Ref. [24]).

In Fig. 3 we display the contact as a function of temperature $T / \varepsilon_{F}$ for different $\ln \left(k_{F} a_{2 \mathrm{D}}\right)$. Over a large temperature range, we observe a very good agreement between the second and the third order of the virial expansion. Moreover, the results from QMC at $T=0$ [14] match our calculated contact reasonably well. However, the expansion starts to break down at low temperatures once we move toward the BCS limit. Interestingly, it appears that the second order of the expansion extrapolates better between the high and low temperature limits than the higher order expansion. As shown in the inset of Fig. 3, the contact is non-monotonic with temperature when $\ln \left(k_{F} a_{2 \mathrm{D}}\right) \gtrsim 1$. This suggests a way of distinguishing the BCS regime from the Bose limit at intermediate temperatures. We speculate that the contact initially increases with temperature in the BCS regime as Pauli blocking becomes less important. For a discussion of non-monotonicity of the 3D contact, see Ref. [25]. In the limit $T \gg \varepsilon_{B}$, we obtain the asymptotic behavior $\mathcal{C} / N \simeq k_{F}^{2} \pi /\left(2 \ln ^{2}\left(a_{2 \mathrm{D}} / \lambda\right)\right)$, which remarkably resembles the $T=0$ weakly interacting limit where $\lambda$ is replaced by $1 / k_{F}$.

Next, we turn to the spectral function $A_{\sigma}(\mathbf{k}, \omega)=$ $-2 \operatorname{Im} G_{\sigma}(\mathbf{k}, \omega)$, which is related to the probability of ex-

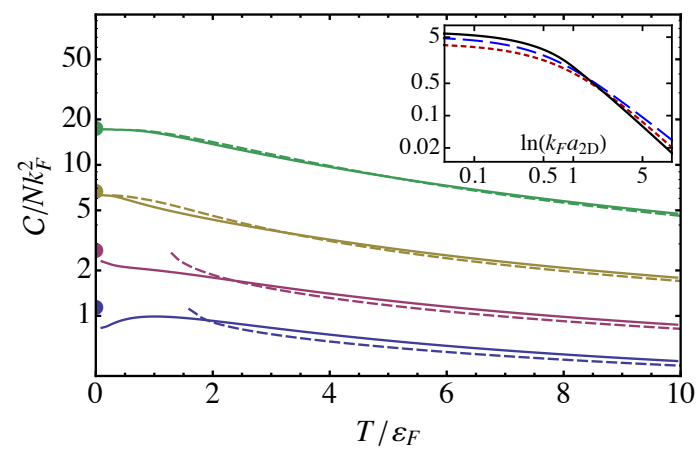

FIG. 3: The contact calculated within the second (solid) and third (dashed) order of the virial expansion for $\ln \left(k_{F} a_{2 \mathrm{D}}\right)=$ $-0.5,0,0.5,1$ (top to bottom). The filled circles are the results of zero-temperature QMC [14]. Inset: Contact from $T=0$ QMC (solid), the virial expansion up to second order at $T=$ $\varepsilon_{F}$ (dashed), and at $T=3 \varepsilon_{F}$ (dotted).

tracting an atom in state $\sigma$ with momentum $\mathbf{k}$ and frequency $\omega$. Thus, it is of great relevance to current experiments measuring the spectral response with momentum resolved photoemission spectroscopy $[5,26]$. The spectral function is obtained from the full Green's function; this is related to the bare propagator via the Dyson equation $G^{-1}=\left[G^{(0)}\right]^{-1}-\Sigma$, where $\Sigma$ is the self-energy. The lowest non-trivial order of the virial expansion yields

$$
\Sigma_{\uparrow}(\mathbf{p}, \tau)={ }_{T_{2}}=z e^{\mu \tau} \sum_{\mathbf{k}} T_{2}(\mathbf{k}, \tau) G_{\downarrow}^{(0,1)}(\mathbf{k}-\mathbf{p},-\tau),
$$

which clearly includes two-body correlations. Considering the occupied part of the spectral function introduces a further power of $z$ from the distribution function, and thus the self energy at this order is equivalent to the second order of the virial expansion. As well as being accurate for $T>\varepsilon_{F}$, our results for the contact suggest that the truncation to second order is also reasonable for $T \simeq \varepsilon_{F}$ when the interaction is not too weak. Indeed, this lowest-order spectral function has been applied to even lower temperatures in 3D [27].

Figure 4 displays a series of spectra for $T / \varepsilon_{F} \gtrsim 1$ and for a range of $\ln \left(k_{F} a_{2 \mathrm{D}}\right)$ similar to that used in the recent 2D photoemission experiments [5]. In Fig. 4(a), we see a two branch structure that resembles that observed in experiment [5]. The lowest incoherent band corresponds to bound dimers while the upper branch corresponds to excited unpaired atoms. A key point is that the spectra all have $\mu<0$ and so the effects of Pauli blocking are minimal. While the temperatures here are higher than those in experiment, we note that all interactions except $\ln \left(k_{F} a_{2 \mathrm{D}}\right)=0.8$, the lowest interaction considered in experiment, have $\mu<0$ at $T=0$. Furthermore, $\ln \left(k_{F} a_{2 \mathrm{D}}\right)=0.8$ only has $\mu \simeq 0.2 \varepsilon_{F}$ at $T=0$ and thus the Fermi gas is no longer degenerate once $T \gtrsim 0.2 \varepsilon_{F}$, i.e., for typical experimental temperatures. Note, fur- 

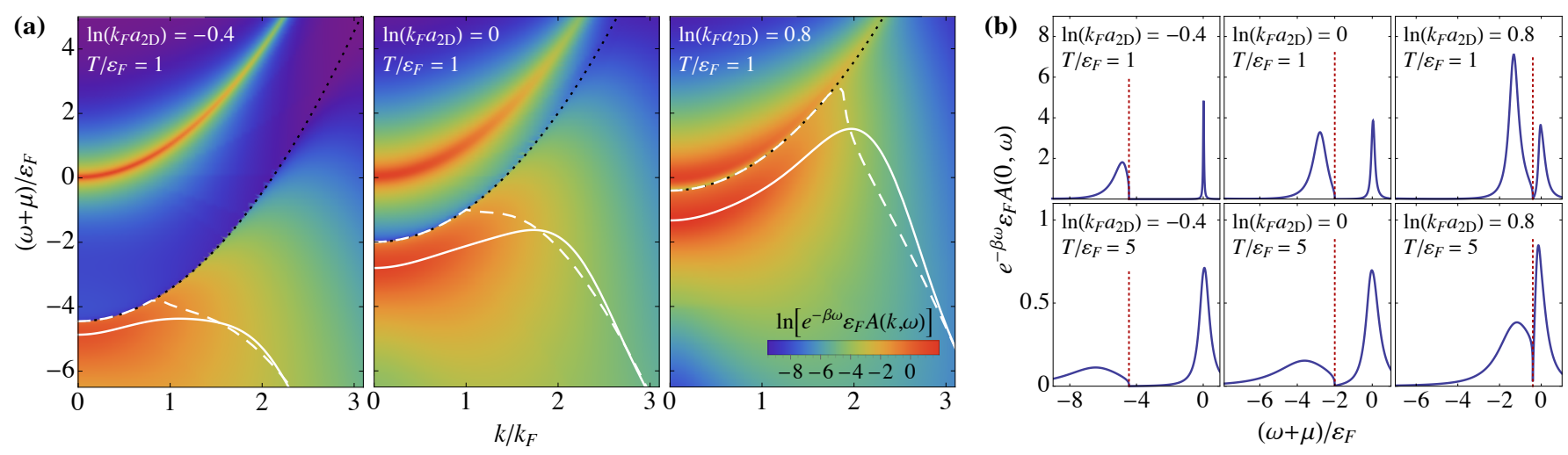

FIG. 4: (a) The occupied part of the spectral function for various values of the interaction parameter $\ln \left(k_{F} a_{2 \mathrm{D}}\right) \operatorname{near}$ the crossover. They all correspond to expansion parameter $z e^{\beta \varepsilon_{B} / 2} \simeq 0.7$. The (black) dotted line corresponds to the free particle dispersion shifted by the binding energy, i.e., $\epsilon_{\mathbf{k}}-\varepsilon_{B}$, while the (white) solid line depicts the position of the peak in the incoherent part of the spectrum at negative energies. The (white) dashed line is the peak position predicted for a thermal gas of bosonic dimers. (b) Slices of the spectra at $\mathbf{k}=0$ for two different temperatures. The vertical dotted lines indicate the two-body binding energy $-\varepsilon_{B}$.

ther, that our statements appear to be unaffected by the presence of a harmonic trapping potential, since we find that the average chemical potential at $T=0$ in the trap also vanishes at $\ln \left(k_{F} a_{2 \mathrm{D}}\right) \simeq 0.5$, where $k_{F}$ is defined as in Ref. [6].

Therefore, our results suggest that the observed pairing gap [5] effectively arises from two-body physics and does not correspond to a pseudogap regime. This view is further supported by the fact that the pairing gap in the spectrum persists to very high temperatures well above $T_{c}$, as shown in Fig. 4(b). Moreover, we see that the "closure" of the gap with increasing temperature appears to be due to the thermal broadening of the two branches.

By examining the peak position of the incoherent branch, we obtain a dispersion that exhibits a "backbending" feature, as shown in Fig. 4(a). A similar feature has been observed in 3D and has been interpreted as evidence for a gapped Fermi surface [28]. However, in our case, it appears to be a consequence of shortrange pairing correlations, similar to what was described in Ref. [29]. Moreover, the momentum at which the backbending occurs is set by $a_{2 \mathrm{D}}$ and $\lambda$ rather than $k_{F}$.

We can compare our results with the atom spectrum obtained for a thermal gas of dimers. Here, one probes the spectrum by exciting one of the atoms within the dimer into a non-interacting state, effectively dissociating the dimer. The resulting (occupied part of the) spectral function is then proportional to

$$
\begin{aligned}
& \frac{\mathcal{C}_{0}}{m^{2}} \Theta\left(\frac{k^{2}}{2 m}-\varepsilon_{B}-(\omega+\mu)\right) \frac{e^{-\beta\left(3 \frac{k^{2}}{2 m}-\varepsilon_{B}-(\omega+\mu)\right)}}{\left(\frac{k^{2}}{2 m}-(\omega+\mu)\right)^{2}} \\
& \times I_{0}\left(2 \beta \frac{k}{\sqrt{m}} \sqrt{\frac{k^{2}}{2 m}-\varepsilon_{B}-(\omega+\mu)}\right)
\end{aligned}
$$

where $I_{0}(x)$ is a modified Bessel function of the first kind, and $\mathcal{C}_{0}=2 \pi N / a_{2 \mathrm{D}}^{2}$ is the two-body contact. This yields a back-bending feature similar to the one obtained in the virial expansion, as shown in Fig. 4(a), and we clearly see how the short-range correlations appear in $A(\mathbf{k}, \omega)$. Note that the incoherence of the lower band is due to the fact that we have a thermal gas of dimers. If they had been condensed, then we would expect a strongly peaked signal at $\omega+\mu=-\varepsilon_{B}-\epsilon_{\mathbf{k}}$.

Finally, we note that the virial coefficients obtained above for the uniform system may be straightforwardly related to those for an isotropic harmonic confinement [21] by means of a local density approximation: in the thermodynamic limit where the temperature is much larger than the trapping frequency $\omega$, we define the local chemical potential $\mu(\rho) \equiv \mu-\frac{1}{2} m \omega^{2} \rho^{2}$, with $\rho$ the radial coordinate in the trap. Then the fugacity also depends on the distance from the center of the trap, $z(\rho) \equiv z \exp \left(-\frac{1}{2} m \omega^{2} \rho^{2} \beta\right)$. Importantly, the virial coefficients are constant over the trap, and the total number of particles in the trap is easily obtained by integrating Eq. (2) to yield $N=\frac{2}{\beta^{2} \omega^{2}} \sum_{j \geq 1} b_{j} z^{j}$. On the other hand, Ref. [21] calculates the thermodynamic potential in the isotropic harmonic trap, $\Omega=-\frac{2}{\beta^{3} \omega^{2}} \sum_{j \geq 1} b_{j}^{\text {trap }} z^{j}$. Computing the particle number $N=-\partial \Omega /\left.\partial \mu\right|_{T, V}$, we identify $b_{j}=j b_{j}^{\text {trap }}$. Using this relation, we conclude that our virial coefficients match exactly those of Ref. [21].

In conclusion, we have investigated the temperature dependence of the contact and the spectral function for the normal 2D Fermi gas. We have argued that current experiments are mainly in the Bose regime and are thus unlikely to have observed pairing that is strongly modified by Fermi statistics. It remains to be seen whether pseudogap phenomena could be observed for weaker interactions in 2D [30]. An unequivocal signature would be the presence of a gap-like feature in the dispersion at 
finite momentum, occurring at the chemical potential. In the future, it would be interesting to consider the mass imbalanced case, where higher partial waves are important in the three-body problem [31] and the third virial coefficient can become non-monotonic [32].

We thank M. Bauer, N. R. Cooper, and X. Leyronas for stimulating discussions. X.-J. Liu, H. Hu, and P. D. Drummond are thanked for sharing their data on the $2 \mathrm{D}$ virial coefficients in a trap. JL acknowledges support from the Carlsberg Foundation and MMP acknowledges support from the EPSRC under Grant No. $\mathrm{EP} / \mathrm{H} 00369 \mathrm{X} / 2$. This work was supported in part by the National Science Foundation under Grant No. PHYS1066293 and the hospitality of the Aspen Center for Physics.

[1] M. Randeria, J.-M. Duan, and L.-Y. Shieh, Phys. Rev. Lett. 62, 981 (1989).

[2] M. Randeria, J.-M. Duan, and L.-Y. Shieh, Phys. Rev. B 41, 327 (1990).

[3] S. Schmitt-Rink, C. M. Varma, and A. E. Ruckenstein, Phys. Rev. Lett. 63, 445 (1989).

[4] K. Martiyanov, V. Makhalov, and A. Turlapov, Phys. Rev. Lett. 105, 030404 (2010).

[5] M. Feld, B. Fröhlich, E. Vogt, M. Koschorreck, and M. Köhl, Nature 480, 75 (2011).

[6] B. Fröhlich, M. Feld, E. Vogt, M. Koschorreck, W. Zwerger, and M. Köhl, Phys. Rev. Lett. 106, 105301 (2011).

[7] P. Dyke, E. D. Kuhnle, S. Whitlock, H. Hu, M. Mark, S. Hoinka, M. Lingham, P. Hannaford, and C. J. Vale, Phys. Rev. Lett. 106, 105304 (2011).

[8] A. T. Sommer, L. W. Cheuk, M. J. H. Ku, W. S. Bakr, and M. W. Zwierlein, Phys. Rev. Lett. 108, 045302 (2012).

[9] Y. Zhang, W. Ong, I. Arakelyan, and J. E. Thomas, Phys. Rev. Lett. 108, 235302 (2012).

[10] S. K. Baur, B. Fröhlich, M. Feld, E. Vogt, D. Pertot, M. Koschorreck, and M. Köhl, Phys. Rev. A 85, 061604 (2012).

[11] M. Koschorreck, D. Pertot, E. Vogt, B. Frohlich, M. Feld, and M. Kohl, Nature 485, 619 (2012).

[12] B. Fröhlich, M. Feld, E. Vogt, M. Koschorreck, M. Köhl,
C. Berthod, and T. Giamarchi, Phys. Rev. Lett. 109, 130403 (2012).

[13] V. Makhalov, K. Martiyanov, and A. Turlapov, arXiv:1305.4411.

[14] G. Bertaina and S. Giorgini, Phys. Rev. Lett. 106, 110403 (2011).

[15] A. J. Leggett, in Modern Trends in the Theory of Condensed Matter, edited by A. Pekalski and J. Przystawa (Springer-Verlag, Berlin, 1980), p. 14.

[16] M. M. Parish, B. Mihaila, E. M. Timmermans, K. B. Blagoev, and P. B. Littlewood, Phys. Rev. B 71, 064513 (2005).

[17] V. Gurarie and L. Radzihovsky, Annals of Physics 322, 2 (2007).

[18] N. Trivedi and M. Randeria, Phys. Rev. Lett. 75, 312 (1995).

[19] V. M. Loktev, R. M. Quick, and S. G. Sharapov, Physics Reports 349, 1 (2001).

[20] S. Tan, Annals of Physics 323, 2971 (2008).

[21] X.-J. Liu, H. Hu, and P. D. Drummond, Phys. Rev. B 82, 054524 (2010).

[22] X. Leyronas, Phys. Rev. A 84, 053633 (2011).

[23] F. Werner and Y. Castin, Phys. Rev. A 86, 013626 (2012).

[24] X.-J. Liu, Physics Reports 524, 37 (2013).

[25] Z. Yu, G. M. Bruun, and G. Baym, Phys. Rev. A 80, 023615 (2009).

[26] J. T. Stewart, J. P. Gaebler, and D. S. Jin, Nature 454, 744 (2008).

[27] H. Hu, X.-J. Liu, P. D. Drummond, and H. Dong, Phys. Rev. Lett. 104, 240407 (2010).

[28] J. P. Gaebler, J. T. Stewart, T. E. Drake, D. S. Jin, A. Perali, P. Pieri, and G. C. Strinati, Nature Phys. 6 (2010).

[29] W. Schneider and M. Randeria, Phys. Rev. A 81, 021601 (2010).

[30] M. Bauer, M. M. Parish, and T. Enss, arXiv:1311.1000.

[31] V. Ngampruetikorn, M. M. Parish, and J. Levinsen, EPL (Europhysics Letters) 102, 13001 (2013).

[32] K. M. Daily and D. Blume, Phys. Rev. A 85, 013609 (2012).

[33] The $T$-matrix in imaginary time is related to the usual frequency-dependent expression $\tilde{T}_{2}(\omega)=$ $-\frac{4 \pi}{m} \frac{1}{\ln \left(-\omega / \varepsilon_{B}\right)}$ via a Laplace transformation $T_{2}(\mathbf{k}, \tau)=$ $\int \frac{d \omega}{2 \pi i} e^{-\omega \tau} \tilde{T}_{2}\left(\omega-\frac{k^{2}}{2 M}\right)$. 